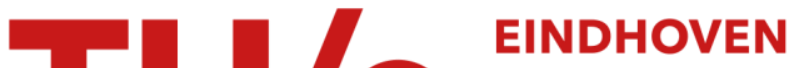 UNIVERSITY OF TECHNOLOGY
}

\section{2-(Diphenylphosphino)phenyl 2-(diphenylphosphinoyl)phenyl ether}

Citation for published version (APA):

Tooke, D. M., Wilting, J. B. M., Vogt, D., \& Spek, A. L. (2005). 2-(Diphenylphosphino)phenyl 2(diphenylphosphinoyl)phenyl ether. Acta Crystallographica, Section E: Structure Reports, E61(8), o2406-02407. https://doi.org/10.1107/S1600536805020805

DOI:

$10.1107 / \mathrm{S} 1600536805020805$

Document status and date:

Published: 01/01/2005

\section{Document Version:}

Publisher's PDF, also known as Version of Record (includes final page, issue and volume numbers)

\section{Please check the document version of this publication:}

- A submitted manuscript is the version of the article upon submission and before peer-review. There can be important differences between the submitted version and the official published version of record. People interested in the research are advised to contact the author for the final version of the publication, or visit the $\mathrm{DOI}$ to the publisher's website.

- The final author version and the galley proof are versions of the publication after peer review.

- The final published version features the final layout of the paper including the volume, issue and page numbers.

Link to publication

\section{General rights}

Copyright and moral rights for the publications made accessible in the public portal are retained by the authors and/or other copyright owners and it is a condition of accessing publications that users recognise and abide by the legal requirements associated with these rights.

- Users may download and print one copy of any publication from the public portal for the purpose of private study or research.

- You may not further distribute the material or use it for any profit-making activity or commercial gain

- You may freely distribute the URL identifying the publication in the public portal.

If the publication is distributed under the terms of Article 25fa of the Dutch Copyright Act, indicated by the "Taverne" license above, please follow below link for the End User Agreement:

www.tue.nl/taverne

Take down policy

If you believe that this document breaches copyright please contact us at:

openaccess@tue.nl

providing details and we will investigate your claim. 
Acta Crystallographica Section E

Structure Reports

Online

ISSN 1600-5368

\section{Duncan M. Tooke, ${ }^{\mathrm{a} *}$ Jos Wilting, ${ }^{b}$ Dieter $\operatorname{Vogt}^{\mathrm{b}}$ and Anthony L. Spek ${ }^{a}$}

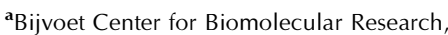
Crystal and Structural Chemistry, Utrecht University, Padualaan 8, $3584 \mathrm{CH}$ Utrecht, The Netherlands, and ${ }^{\mathbf{b}}$ Schuit Institute of Catalysis, PO Box 513, 5600 MB Eindhoven,

The Netherlands

Correspondence e-mail: d.m.tooke@chem.uu.nl

Key indicators

Single-crystal X-ray study

$T=150 \mathrm{~K}$

Mean $\sigma(\mathrm{C}-\mathrm{C})=0.002 \AA$

$R$ factor $=0.039$

$w R$ factor $=0.098$

Data-to-parameter ratio $=18.1$

For details of how these key indicators were automatically derived from the article, see http://journals.iucr.org/e.

\section{2-(Diphenylphosphino)phenyl 2-(diphenyl- phosphinoyl)phenyl ether}

The title compound, $\mathrm{C}_{36} \mathrm{H}_{28} \mathrm{O}_{2} \mathrm{P}_{2}$, features weak inter- and intramolecular hydrogen bonds linking molecules into infinite chains.

\section{Comment}

The title compound, (I), was inadvertently obtained during an attempt to synthesize a nickel-phosphine complex.<smiles>O=P(c1ccccc1)(c1ccccc1)c1ccccc1Oc1ccccc1P(c1ccccc1)c1ccccc1</smiles>

(I)

The structure features a weak hydrogen bond between aromatic atom $\mathrm{H} 6$ and the phosphine oxide $\mathrm{O}$ atom, which a search of the Cambridge Structural Database (Version 5.26; Allen, 2002) shows to be a common feature in phenyl-substituted phosphine oxides. An additional weak (Steiner, 1996) bifurcated intermolecular hydrogen bond is also present between $\mathrm{O} 2$ and $\mathrm{H} 27$ and $\mathrm{H} 28$, which joins the molecules into an infinite chain along [1̄ㅣ․

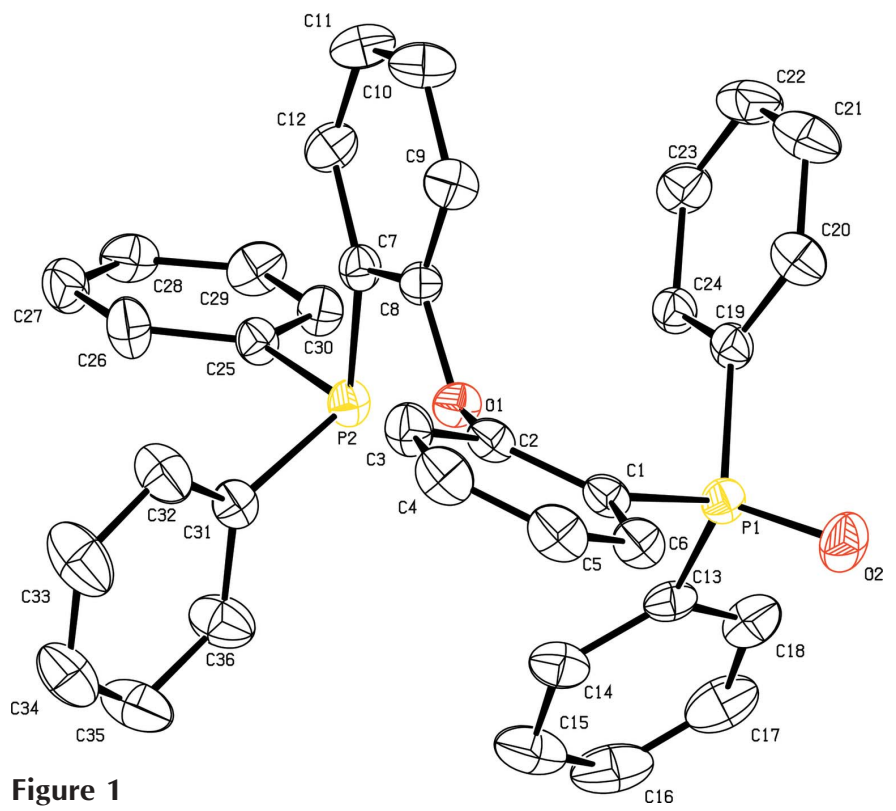

View of the title compound with the atom-numbering scheme. Displacement ellipsoids are drawn at the $50 \%$ probability level. $\mathrm{H}$ atoms have been omitted for clarity.
(C) 2005 International Union of Crystallography Printed in Great Britain - all rights reserved
Received 29 June 2005 Accepted 30 June 2005 Online 6 July 2005 


\section{Experimental}

A tetrahydrofuran solution of 2 equivalents of bis[2-(diphenylphosphino)phenyl] ether and bis(1,5-cyclooctadiene)nickel(0) was layered with $n$-pentane and placed in a freezer, resulting in a crop of off-white crystals after 5 d. ${ }^{31} \mathrm{P}$ NMR $\left(162 \mathrm{MHz}, \mathrm{C}_{6} \mathrm{D}_{6}\right): \delta 22.8(s)$, $-17.5(s)$.

\section{Crystal data}

$\mathrm{C}_{36} \mathrm{H}_{28} \mathrm{O}_{2} \mathrm{P}_{2}$

$M_{r}=554.52$

Triclinic, $P \overline{1}$

$a=9.9316(7) \AA$

$b=10.2786(5) \AA$

$c=14.5778(10) \AA$

$\alpha=75.785(4)^{\circ}$

$\beta=83.778(6)^{\circ}$

$\gamma=85.529(6)^{\circ}$

$V=1432.05(16) \AA^{3}$

$$
\begin{aligned}
& Z=2 \\
& D_{x}=1.286 \mathrm{Mg} \mathrm{m}^{-3} \\
& \text { Mo } K \alpha \text { radiation } \\
& \text { Cell parameters from } 152 \\
& \quad \text { reflections } \\
& \theta=4.3-21.9^{\circ} \\
& \mu=0.18 \mathrm{~mm}^{-1} \\
& T=150(2) \mathrm{K} \\
& \text { Block, colourless } \\
& 0.3 \times 0.3 \times 0.15 \mathrm{~mm}
\end{aligned}
$$

Data collection

Nonius KappaCCD diffractometer $\omega$ and $\varphi$ scans

$R_{\text {int }}=0.044$

$\theta_{\max }=27.5^{\circ}$

$h=-12 \rightarrow 12$

$k=-13 \rightarrow 13$

$l=-18 \rightarrow 18$

6527 independent reflections

5195 reflections with $I>2 \sigma(I)$

Refinement

Refinement on $F^{2}$

$R\left[F^{2}>2 \sigma\left(F^{2}\right)\right]=0.039$

$w R\left(F^{2}\right)=0.098$

$S=1.04$

6527 reflections

361 parameters

$\mathrm{H}$-atom parameters constrained

$$
\begin{gathered}
w=1 /\left[\sigma^{2}\left(F_{\mathrm{o}}{ }^{2}\right)+(0.0396 P)^{2}\right. \\
+0.7162 P] \\
\text { where } P=\left(F_{\mathrm{o}}{ }^{2}+2 F_{\mathrm{c}}{ }^{2}\right) / 3 \\
(\Delta / \sigma)_{\max }=0.001 \\
\Delta \rho_{\max }=0.31 \mathrm{e}^{-3} \\
\Delta \rho_{\min }=-0.33 \AA^{-3}
\end{gathered}
$$

\section{Table 1}

Hydrogen-bond geometry $\left(\AA{ }^{\circ}\right)$.

\begin{tabular}{lllll}
\hline$D-\mathrm{H} \cdots A$ & $D-\mathrm{H}$ & $\mathrm{H} \cdots A$ & $D \cdots A$ & $D-\mathrm{H} \cdots A$ \\
\hline $\mathrm{C} 6-\mathrm{H} 6 \cdots \mathrm{O} 2$ & 0.95 & 2.56 & $2.975(2)$ & 107 \\
$\mathrm{C} 27-\mathrm{H} 27 \cdots \mathrm{O} 22^{\mathrm{i}}$ & 0.95 & 2.59 & $3.206(2)$ & 123 \\
$\mathrm{C} 28-\mathrm{H} 28 \cdots \mathrm{O} 22^{\mathrm{i}}$ & 0.95 & 2.58 & $3.202(2)$ & 123 \\
\hline
\end{tabular}

Symmetry code: (i) $x-1, y+1, z$.

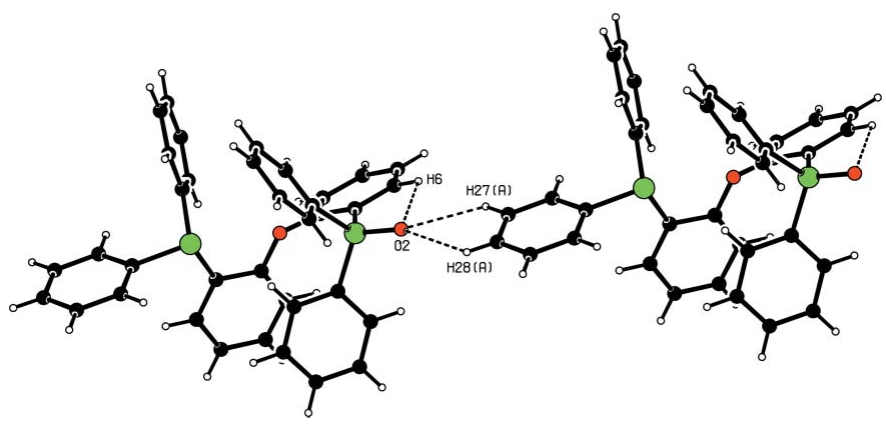

Figure 2

$\mathrm{C}-\mathrm{H} \cdots \mathrm{O}$ hydrogen bonding (dashed lines) around the phosphine oxide. [Symmetry code: (A) $1+x, 1-y, z$.

All $\mathrm{H}$ atoms were placed in geometrically idealized positions $(\mathrm{C}-$ $\mathrm{H}=0.95 \AA$ ) and constrained to ride on their parent atoms, with $U_{\text {iso }}(\mathrm{H})=1.5 U_{\text {eq }}(\mathrm{C})$ for methyl $\mathrm{H}$ atoms and $U_{\text {iso }}(\mathrm{H})=1.2 U_{\text {eq }}(\mathrm{C})$ for all other $\mathrm{H}$ atoms.

Data collection: COLLECT (Hooft, 1998); cell refinement: DIRAX (Duisenberg, 1992); data reduction: EVALCCD (Duisenberg et al., 2003); program(s) used to solve structure: SHELXS86 (Sheldrick, 1985); program(s) used to refine structure: SHELXL97 (Sheldrick, 1997); molecular graphics: PLATON (Spek, 2003); software used to prepare material for publication: PLATON.

The authors thank the Council for the Chemical Sciences of the Netherlands Organization for Scientific Research (CWNWO) for their support.

\section{References}

Allen, F. H. (2002). Acta Cryst. B58, 380-388.

Duisenberg, A. J. M. (1992). J. Appl. Cryst. 25, 92-96.

Duisenberg, A. J. M., Kroon-Batenburg, L. M. J. \& Schreurs, A. M. M. (2003). J. Appl. Cryst. 36, 220-229.

Hooft, R. W. W. (1998). COLLECT. Nonius BV, Delft, The Netherlands. Sheldrick, G. M. (1985). SHELXS86. University of Göttingen, Germany. Sheldrick, G. M. (1997). SHELXL97. University of Göttingen, Germany. Spek, A. L. (2003). J. Appl. Cryst. 36, 7-13. Steiner, Th. (1996). Crystallogr. Rev. 6, 1-57. 